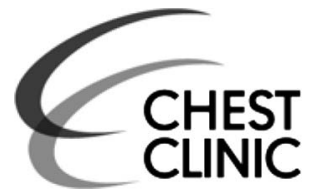
CLIEST

aculty of Medicine of Sousse, Medical Intensive Care Unit, Sahloul University Hospital, Sousse, Tunisia

${ }^{2}$ Faculty of Medicine Ibn El Jazzar, Pharmacology Department, Sousse, Tunisia

\section{Correspondence to} Professor Houssem Hmouda, Faculty of Medicine of Sousse, Medical Intensive Care Unit, Sahloul University Hospital, Route de la ceinture, Sousse 4054, Tunisie; houssem. hmouda1@gmail.com

Received 19 March 2015 Revised 29 May 2015 Accepted 8 June 2015 Published Online First 29 June 2015 2015;70:1004-1006.

\title{
A case-based discussion from the Medical Intensive Care Unit of Sahloul University Hospital of Tunisia: an unusual cause of alveolar hypoventilation in a patient with COPD
}

\author{
Olfa Mejri, ${ }^{1}$ Olfa Beji, ${ }^{1}$ Chaker Ben Salem, ${ }^{2}$ Houssem Hmouda ${ }^{1}$
}

\section{OM (RESIDENT, INTENSIVIST)}

A 54-year-old heavy smoker with a 20-year history of COPD, confirmed by spirometry, and previous gastric ulcer presented to the emergency room with mild mid-epigastric pain, nausea and vomiting evolving over 3 days.

He reported taking a traditional herbal tea made of Retama raetam (white weeping broom) (approximately $1000 \mathrm{~mL}$ ) which caused subsequent symptom deterioration and acute confusion (figure 1).

On examination, the patient had no fever and was confused with a Glasgow coma score of 13/15. His abdomen was soft. The remainder of the physical examination was unremarkable. Chest X-ray, ECG, CT brain scan and laboratory blood tests were normal. Arterial blood gas (ABG) analysis on room air disclosed severe respiratory acidosis with hypercapnia and high bicarbonate level $(\mathrm{pH}=7.14$, $\left.\mathrm{pCO}_{2}=11 \mathrm{kPa}, \mathrm{HCO}_{3}^{-}=36 \mathrm{mmol} / \mathrm{L}\right)$ as well as severe hypoxaemia $\left(\mathrm{paO}_{2}=5.4 \mathrm{kPa}\right)$.

Subsequently, because of a rapid deterioration of the level of consciousness, as assessed by a fall in Glasgow coma score (GCS) to 6/15, the patient required intubation, sedation and mechanical ventilation. His blood pressure and pulse fell substantially soon after he was placed on the ventilator, requiring fluid resuscitation and continuous infusion of norepinephrine $(1 \mathrm{mg} / \mathrm{h})$. The patient was then transferred to the intensive care unit (ICU).

In the ICU, he was maintained on volume assist control ventilation with the following settings: tidal volume $420 \mathrm{~mL}, \mathrm{FIO}_{2} 50 \%$, positive end expiratory pressure (PEEP) $4 \mathrm{~cm} \mathrm{H}_{2} \mathrm{O}$, respiratory rate $14 / \mathrm{min}$ and inspiratory time (I)/expiratory time (E) ratio of $1 / 3$. Pupils were dilated and responsive to light. Invasive arterial blood pressure (IABP) was 120/65/80 mm Hg, respectively, for systolic, diastolic and mean IABPs. Oxygen saturation by pulse oximetry was $100 \%$, and heart rate was around $55 \mathrm{bpm}$. ECG showed sinus bradycardia with no other abnormalities. ABG analysis revealed baseline hypercapnia $\left(\mathrm{pCO}_{2}\right.$ of $\left.6.7 \mathrm{kPa}\right)$ and compensated respiratory acidosis. Blood tests showed normal white cell count, and normal $\mathrm{C}$ reactive protein and procalcitonin levels. Portable chest X-ray showed bilateral basal haziness, but chest CT scan was normal. Further investigations, including abdominal CT scan and ultrasound, were normal. Echocardiography showed normal left and right ventricular function, and pulmonary arterial pressure was estimated as $30 \mathrm{~mm} \mathrm{Hg}$. Microbiological investigations, including protected bronchial distal aspirate, and blood cultures were negative. Urine toxicology testing revealed the presence of quinolizidine alkaloid metabolites.

The patient remained stable on mechanical ventilation. Norepinephrine was stopped on the third day. Weaning from mechanical ventilation was attempted on the fourth day, with successful extubation and the patient was discharged from ICU on the sixth day. He was seen 1 week later in the outpatient clinic and was in a remarkably good physical condition.

\section{OB (ASSISTANT PROFESSOR, INTENSIVIST)}

Alveolar hypoventilation is a frequent cause of acute worsening of gas exchange in patients with COPD. It should be distinguished from COPD exacerbations caused, or triggered, by a variety of factors, including viral or bacterial lower airways infection, or air pollution. ${ }^{1-3}$ In one-third of all exacerbations, the cause cannot be identified. ${ }^{4}$ Careful ABG analysis assists understanding of the mechanism of the acute deterioration in the respiratory condition.

\section{OM (RESIDENT, INTENSIVIST)}

How would we determine the mechanism of gas exchange alteration in our patient?

\section{HH (PROFESSOR, INTENSIVIST)}

Exacerbations of COPD are characterised by a worsening of pulmonary gas exchange with increased alveolar-arterial oxygen gradient (A-a gradient or A-a $\mathrm{O}_{2}$ ).

A-a gradient is a measure of the difference between the alveolar concentration (A) of oxygen and the arterial (a) concentration of oxygen.

A-a gradient $=\mathrm{pAO}_{2}-\mathrm{paO}_{2}$, where $\mathrm{pAO}_{2}$ is alveolar $\mathrm{pO}_{2}$ calculated from the alveolar gas equation: $\mathrm{pAO}_{2}=\mathrm{FiO}_{2}\left(\right.$ patm $\left.-\mathrm{pH}_{2} \mathrm{O}\right)-\mathrm{paCO}_{2} / \mathrm{R}$, and $\mathrm{paO}_{2}$ is the arterial $\mathrm{paO}_{2}$ measured in arterial blood and $\mathrm{R}$ is the respiratory exchange ratio.

On room air $\left(\mathrm{FiO}_{2}=21 \%\right)$, at sea level (patm $=760 \mathrm{~mm} \mathrm{Hg}$ ), assuming 100\% humidity in the alveoli (water vapour pressure of $6.3 \mathrm{kPa}$ ) and $\mathrm{R}=0.8$, the equation can be simplified: A-a gradient $=\left(20-\mathrm{paCO}_{2} / 0.8\right)-\mathrm{PaO}_{2}$. A-a gradient calculation is facilitated by on-line calculators. 


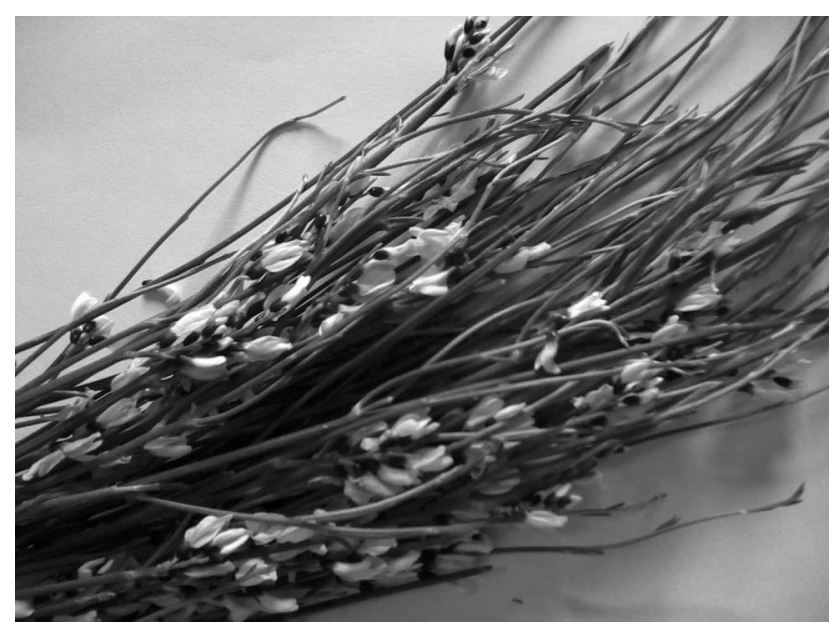

Figure 1 The medicinal plant Retama raetam.

Careful determination of A-a gradient is a valuable tool for understanding the mechanism of hypoxaemia: either pulmonary or extrapulmonary.

In a young non-smoker adult breathing room air, A-a gradient is between 0.7 and $1.3 \mathrm{kPa}(5-10 \mathrm{~mm} \mathrm{Hg})$. It is expected to increase by $0.13 \mathrm{kPa}(1 \mathrm{~mm} \mathrm{Hg})$ every decade. Normal A-a gradient is estimated to be less than (age in years/4) +4 (divide by 7.5 for $\mathrm{kPa}$ ).

A-a gradient increases with age, reflecting the slight decline in $\mathrm{paO} 2$ due to change in ventilation perfusion ratio (VA/Q) of the ageing lungs.

\section{OM (RESIDENT, INTENSIVIST)}

In this case, calculated $\mathrm{A}$-a gradient is $0.8 \mathrm{kPa}$, which is within the normal range. How does this help in deeper discussion of the case?

\section{HH (PROFESSOR, INTENSIVIST)}

In daily practice, a pathophysiologically based discussion of the differential diagnosis of hypoxaemia is highly recommended for rational management. Indeed, in the present case, the normal A- a gradient was helpful in ruling out several mechanisms of hypoxaemia and their causes:

1. Ventilation perfusion inequality $(\mathrm{VA} / \mathrm{Q}<1)$ and intrapulmonary shunt $(\mathrm{VA} / \mathrm{Q}=0)$ due to pneumonia, atelectasis, cardiogenic pulmonary oedema, pulmonary infarction secondary to acute PE and acute respiratory distress syndrome were excluded. They were not suggested by the initial clinical presentation and were ruled out by laboratory investigations. The normal A-a gradient was a strong argument against this mechanism, as well as the favourable outcome with volume controlled mechanical ventilation without the need for either increasing inspired oxygen fraction $\left(\mathrm{FIO}_{2}\right)$ or setting a high level of PEEP.

2. Right to left extrapulmonary shunt, particularly an intracardiac shunt, due to a patent foramen ovale should be considered in the setting of COPD and corpulmonale. It was ruled out by echocardiography. In this setting, supplemental oxygen does not improve hypoxaemia.

3. Diffusion impairment is another cause of an elevated A-a gradient, but only during exercise in the context of an interstitial lung disease, and $\mathrm{paCO}_{2}$ is usually normal, none of which applies in our patient. In the case of pulmonary fibrosis, supplemental oxygen produces substantial improvement in hypoxaemia.
4. Alveolar hypoventilation where the A-a gradient is normal, and $\mathrm{paCO}_{2}$ is elevated was the appropriate mechanism of hypoxaemia in our patient. The favourable outcome with mechanical ventilation supports this mechanism. A broad range of aetiologies can produce alveolar hypoventilation; they include muscular weakness, diaphragmatic fatigue, severe hypophosphataemia, diseases of the neuromuscular junction, diseases of nerves supplying respiratory muscles, brainstem disease and drug-induced central nervous system depression.

5. It should be kept in mind that it is not rare to have mixed causes of hypoxaemia, and that, in the acutely ill, it is sometimes difficult to assess with accuracy the contribution of each mechanism.

\section{OB (ASSISTANT PROFESSOR, INTENSIVIST)}

Our patient had a normal A-a gradient, suggesting an extrapulmonary origin of his respiratory failure. Furthermore, common causes of acute COPD exacerbation were ruled out. There was no evidence of pulmonary or extrapulmonary infection; chest CT scan and transthoracic echocardiography ruled out acute PE as well as left ventricular failure. Blood tests showed no metabolic disorders.

\section{OM (RESIDENT, INTENSIVIST)}

Could Retama raetam tea ingestion possibly be the cause of alveolar hypoventilation in our patient?

\section{CBS (ASSOCIATE PROFESSOR, PHARMACOLOGIST)}

Retama raetam herbal tea ingestion was almost certainly the cause of the acute deterioration of the clinical condition with alveolar hypoventilation secondary to central nervous system and respiratory depression. Indeed, Retama raetam is known for its quinolizidine alkaloid properties and for its pharmacological and toxicological activity with diuretic, hypoglycaemic, hypotensive, hallucinogenic and respiratory depressant effects. ${ }^{5} 6$ The clinical presentation of our patient, the absence of the usual causes of COPD exacerbation, the presence of quinolizidine alkaloid metabolites in the urine and the spontaneous favourable outcome with symptomatic treatment alone (mechanical ventilation, vasoactive agents and fluid resuscitation), without any specific therapy, are strong arguments supporting our hypothesis. This could also explain the short time required for mechanical ventilation as well as successful weaning.

\section{HH (PROFESSOR, INTENSIVIST)}

In conclusion, alveolar hypoventilation is an important cause of morbidity and mortality in COPD. Early recognition of the aetiology of hypoventilation is crucial for adequate management and better outcome. A thorough, careful analysis of ABG, including A-a gradient calculation, allows better understanding of the mechanism of hypoxaemia. It should be remembered that A-a gradient calculation is a component of the Acute Physiology and Chronic Health Evaluation (APACHE II) score, routinely determined in most ICUs to predict the severity of critical illness.

Herbal extracts such as quinolizidine alkaloids can induce severe acute hypoventilation in patients with COPD causing hypercapnia and severe hypoxaemia with a normal A-a gradient, requiring mechanical ventilation for a short period of time. This unusual cause of severe hypoventilation should be kept in mind, particularly in the North African and Mediterranean regions, where herbal medicine has a widespread use. 


\section{Chest clinic}

\section{Competing interests None declared.}

Provenance and peer review Not commissioned; externally peer reviewed.

\section{REFERENCES}

1 Mannino DM, Buist AS. Global burden of COPD: risk factors, prevalence, and future trends. Lancet 2007;370:765-73.

2 Vestbo J, Hurd SS, Agusti AG, et al. Global strategy for the diagnosis, management, and prevention of chronic obstructive pulmonary disease: GOLD executive summary. Am J Respir Crit Care Med 2013;187:347-65.
3 Pauwels R, Calverley P, Buist AS, et al. COPD exacerbations: the importance of a standard definition. Respir Med 2004;98:99-107.

4 Bhowmik A, Seemungal TAR, Sapsford RJ, et al. Relation of sputum inflammatory markers to symptoms and lung function changes in COPD exacerbations. Thorax 2000;55:114-200.

5 Maghrani M, Lemhadri A, Jouad $\mathrm{H}$, et al. Effect of the desert plant Retama raetam on glycaemia in normal and streptozotocin-induced diabetic rats. J Ethnopharmacol 2003;87:21-5.

6 Algandaby MM, Alghamdi HA, Ashour OM, et al. Mechanisms of the antihyperglycemic activity of Retama raetam in streptozotocin-induced diabetic rats. Food Chem Toxicol 2010;48:2448-53. 